\title{
DECISION SUPPORT SYSTEM FOR GENERATING ERGONOMIC TOOL-HANDLES
}

\author{
Harih, G. \\ Laboratory for intelligent CAD systems, University of Maribor, \\ Faculty of Mechanical Engineering, Smetanova 17, SI-2000 Maribor, Slovenia \\ E-Mail: gregor.harih@um.si
}

\begin{abstract}
Tool-handle design research has been previously limited to determination of diameters of cylindrical handles to increase performance, comfort and avoid acute and cumulative traumatic disorders. However, there is still lack of correct shape determination and systemization of the ergonomic design knowledge. To overcome these limitations, methodology to develop optimal sized and shaped toolhandles for a target population previously developed has been integrated into an existing ergonomics and aesthetics decision support system. The system allows a correct determination of tool-handle size and shape according to the target population and provides general ergonomics knowledge. Resulting handles consider optimal diameters for each finger to maximize maximum voluntary contraction, comfort and contact area, which can lower the risk of acute and cumulative trauma disorders. The system allows development of ergonomic tool-handles with almost no prior ergonomics knowledge and without iterative design process, which decreases the designing time.

(Received in August 2012, accepted in July 2013. This paper was with the authors 2 months for 2 revisions.)
\end{abstract}

Key Words: Ergonomics, Tool-Handle, Material Choice, Finite Element Method, Decision Support System

\section{INTRODUCTION}

If the product will be human operated, the designer has to consider ergonomics in order to achieve the expected system efficiency [1]. Since most of the products are designed to be human hand operated, many authors have researched the topic of tool-handle design to define the optimal size and shape of a tool-handle. A correctly designed handle can provide safety, comfort and increased performance. Different authors used different criteria to determine optimal cylindrical handle: subjective comfort rating [2, 3]; finger force measurement $[4,5]$; muscle force minimization [6] and hand anthropometrics [2, 7-12]. Few studies also used two or more criteria: finger force measurement and muscle activity [7, 10, 13]; subjective comfort rating, finger force measurement and electromyographic efficiency of muscle activity [14].

Most of the authors have focused on cylindrical or elliptical shapes of the handles. However none of them considered the anatomical shape of the hand in the optimal power grasp posture. It has been shown that handles should vary in size between hand and finger size, since maximum possible exerted finger force is diameter dependent [14]. It has been also shown that objects that follow the shape of the hand result in much lower local contact pressures, which can prevent discomfort and several disorders [15]. Authors suggested that further research of this topic should consider the shape of the hand in the optimal power grasp posture since it could improve the ergonomics of the tool-handle [11].

The mechanical behaviour of the skin and subcutaneous tissue is crucial during gripping tasks, since forces and moments are transferred from the tool to the whole hand-arm system. Skin and subcutaneous tissue have non-linear viscoelastic properties, where the skin is stiffer than the subcutaneous tissue [16]. A power-grasp produces a very uneven distribution of forces and, therefore, also pressure on the hand and fingers [17]. It has been shown, that any higher contact pressures than allowed for over a specific time can result in discomfort, pain, 
and acute traumatic disorders (ATD) and also cumulative traumatic disorders (CTD) [18, 19]. Hand-handle contact-force and pressure are also handle diameter dependant [20, 21]. In [21] authors have shown that the higher grip and push-forces on a cylindrical handle can produce concentrated contact-forces and pressures that exceed the limit of pressure discomfort and sustained pressure values for preserving work efficiency over a working day.

In [22] authors showed that foam rubber grip can be used to provide more uniform distribution of forces and pressures. It has been also shown that rubber as tool-handle material increases the maximum torques from the hand to the handle [23]. This is due to greater frictional coupling between hand-handle interface. Finite element method (FEM) or finite element analysis (FEA) is used to predict the behaviour of observed structure considering particular load and boundary conditions $[24,25]$ and can be used also for wide range of simulations from common structural problems to very specific problems (e.g. tool-wear, biomass production, welding, safety, etc.) $[26,27]$. Therefore it can be also used to simulate the interaction between human fingertip with a tool-handle [15, 28]. In [29] authors showed that FEM can be also used to analyse the contact stiffness between a finger and object when wearing air-cushioned gloves. Material choice in the design phase of a tool-handle has been also modelled, compared and evaluated [30]. It has been already shown, that cellular foam materials can effectively reduce the contact pressure, since hyper-elastic foam materials, which take into account the non-linear behaviour of fingertip soft tissue, can lower the contact pressure whilst maintaining low deformation rate of the tool-handle material for maintaining sufficient rate of stability of the hand tool in the hands.

As presented, tool-handle ergonomics is an interdisciplinary science, therefore designer has to poses wide range of knowledge and also experience to allow a holistic design approach for reaching the expected human-product performance and safety [31]. This extensive ergonomic knowledge that is necessary during the design phase of a tool-handle; and its poor integration with existing, well-established Computer Aided Design (CAD) software, has affected companies that do not or on very low scales address ergonomic principles during the design phase [31]. In order to overcome this issue, many authors have developed ergonomic aid tools.

Modern product development is heavily CAD based, therefore authors tried to develop ergonomic analysis tools, which can be integrated into the three-dimensional modelling design phase of the product [32]. These systems try to consider important ergonomic principles in the design phase of the product and allow performing ergonomic analyses inside the CAD software for a streamlined workflow. However these systems usually consist of general ergonomic recommendations and do not provide biomechanically correct human models to model the size and shape of the developing workplace or the product.

To overcome this issue several authors developed digital-human models (DHM) for workplace and also product design. Few authors also developed stand-alone anatomicallyaccurate Digital Human Hand Models (DHHMs) for ergonomic evaluation of hand-held products $[33,34]$. These DHHMs are designed for ergonomic analyses of existing virtual 3D models of products; therefore designers still have to possess comprehensive knowledge of ergonomics in order to lower the design iterations and to obtain a product containing the desired ergonomics. DHHMs do not allow for direct development of the product's shape and size that is within their interactions with the humans.

Therefore a static DHHM in its optimal power-grasp posture for directly modelling a corresponding tool-handle with improved ergonomics was already developed in previous research [35]. It has been shown, that tool-handles developed based on the DHHM increases maximum voluntary contraction of fingers, maximizes the contact area, and, thereby lowers the local and overall contact pressures, and increases the subjective comfort-rating. However the DHHM does not consist of basic ergonomic knowledge for the tool-handle design. 
The aim of this paper was to overcome those limitations of current mathematical models regarding the tool-handle size determination, decision support systems for ergonomic product design and DHHMs that require extensive ergonomic knowledge and iterative design process. Thus previously introduced methods for the developing of anatomically correct DHHM for tool-handle design were integrated into a comprehensive intelligent decision support system for ergonomic design of products called OSCAR [31, 36-38]. The system allows the designer a correct determination of tool-handle size and shape according to the target population and provides also general knowledge regarding tool-handle ergonomics. Additionally, the system provides aid to determine appropriate tool-handle material in order to lower the contact pressure. The resulting tool-handles increase comfort rating, maximize voluntary contraction of fingers, maximize contact area and therefore lower the local and overall contact pressure. Thereby the user's performance can be increased and the chance for fatigue and acute and also cumulative trauma disorders can be lowered. Therefore the whole system allows the designers development of products with improved ergonomics in shorter time.

\section{METHODS}

\subsection{Determination of optimal sized and shaped tool-handle}

Based on the traditional design approach to determine optimal diameter of a tool-handle, a medical approach to obtain the shape of the subject's hand in its optimal power grasp posture was developed [39]. Tool-handle was then modelled based on the obtained 3D hand model using traditional CAD modelling techniques as summarized hereafter.

A $50^{\text {th }}$ percentile male subject according to ANSUR database [40] with no hand injuries or and other hand disorders was used to determine the optimal sized and shaped tool-handle. Most recent studies to determine the optimal handle diameters were considered in this paper $[11,14,41]$. Anthropometric measurements of the subject were performed on the subject's hand. Afterwards optimal diameters for each finger were calculated, which were used to manufacture an optimal pre-handle with variable diameters. To maintain the shape of the hand in its optimal power grasp posture, an outer hand mould was manufactured using orthotic material while softly holding the optimal pre-handle. Afterwards magnetic resonance imaging (MRI) with slice thickness of $1 \mathrm{~mm}$ was performed using a Signa HDxt 3.0T MRI machine with outer hand mould attached to hand to maintain the optimal power grasp posture. Obtained images in DICOM file format were used to perform segmentation and 3D reconstruction in professional medical imaging and editing software Amira ${ }^{\circledR}$ 5.3.3. (Visage Imaging $^{\circledR}$ ). The obtained 3D model of the subject's hand was then imported into reverse engineering software Geomagic Studio ${ }^{\circledR}$ 2012. An elliptical cylinder was modelled and positioned in the empty palmar side of the hand. The anatomically sized and shaped toolhandle was then obtained using Boolean operation, which removed the volume of the handle that was in overlap with the hand. Thereby a tool-handle with best fit could be obtained (Fig. $1)$.

\subsection{Creating a symmetrical handle}

The obtained tool-handle was edited in order to obtain a symmetrical handle, which allows usage with both hands. Therefore a cutting/mirroring plane was modelled. The position of the cutting plane was determined to maintain the optimal diameters for each finger of the resulting symmetrical tool-handle. Thereby also all benefits of correct diameter determination could be maintained (maximum voluntary contraction, diameter dependent comfort rating, etc). Afterwards the plane was used to cut the thumb side of the handle. Then the same plane 
was used to apply a mirroring function of the remaining handle shape to obtain a symmetrical handle (Fig. 2). Low smoothing was applied locally to correct small topological irregularities. When creating a symmetrical handle, the optimal shape of the handle is lost due to the mirroring process. However, lost is only the thumb groove, which has not substantial proportion to the performance and comfort. Following has been also tested and observed using rapid prototypes and test subjects [35].

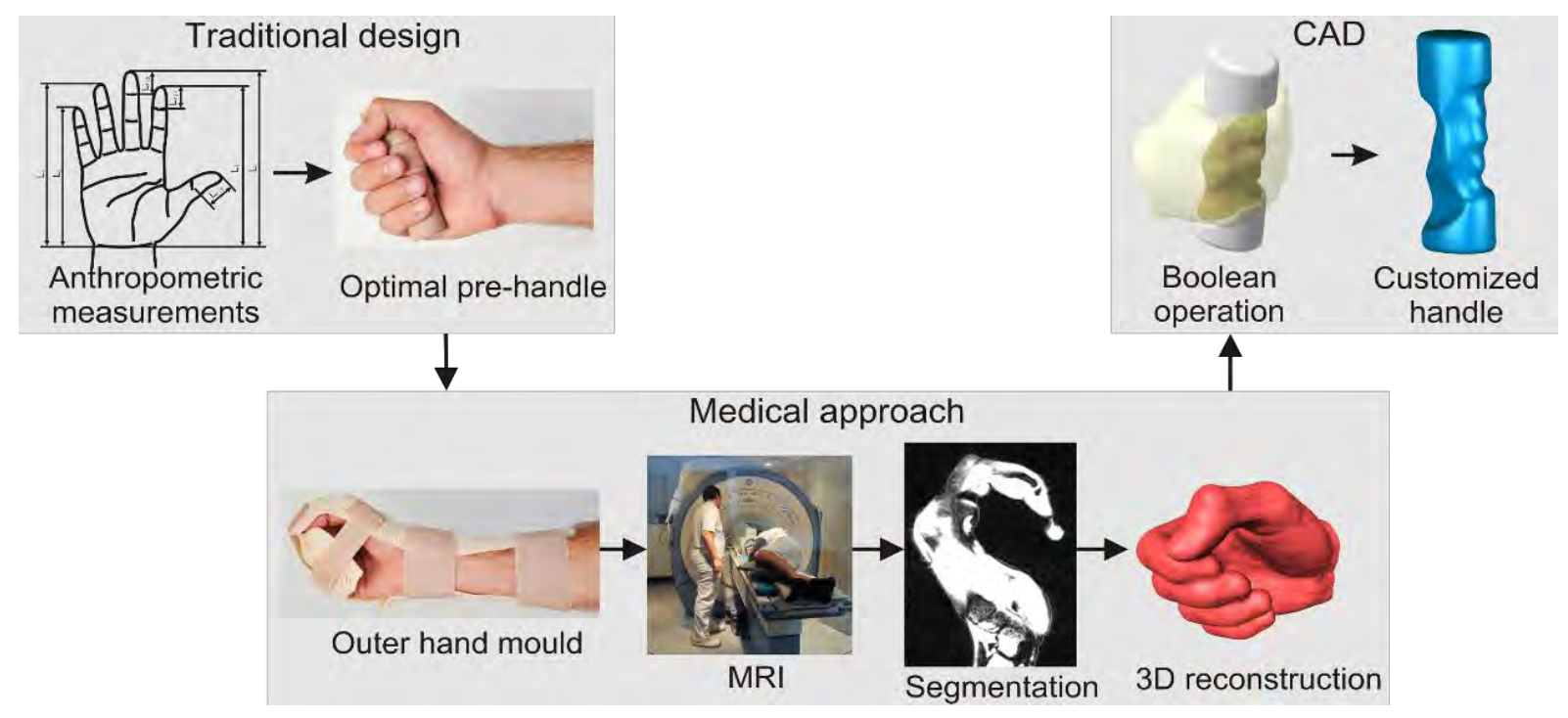

Figure 1: Used methods for obtaining optimal sized and shaped tool-handle.

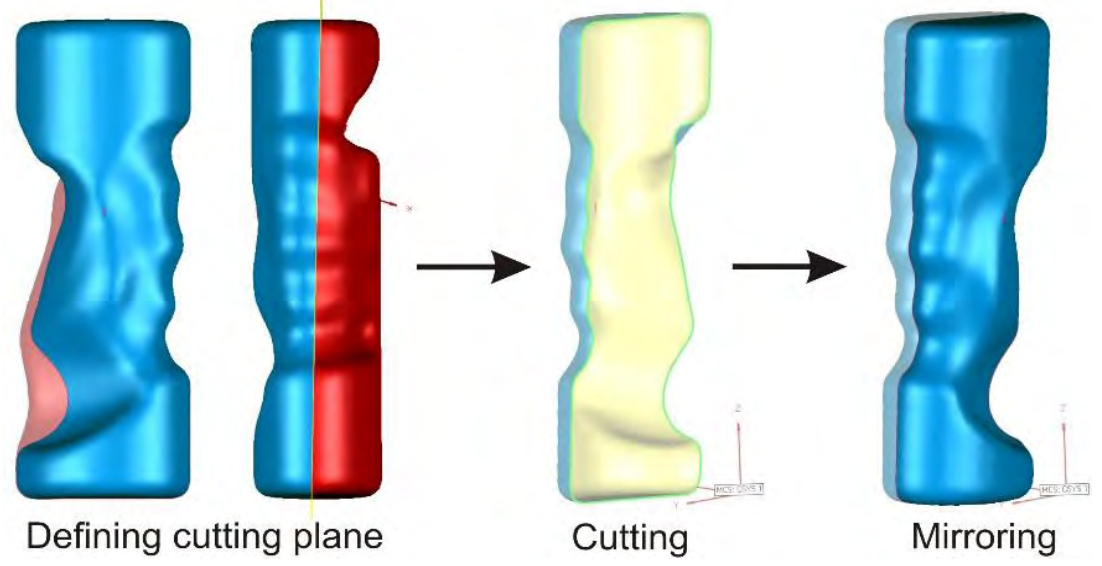

Figure 2: Creating a symmetrical tool-handle.

\subsection{Sizing the tool-handle for target populations}

In order to obtain a tool-handle for different target populations, scaling functions are introduced. Based on analysis performed by [34], it has been shown that the most variation of the anthropometric measurements of the human hand can be attributed to two main anthropometric measurements, which represent hand width and hand length. Additionally, the hand length measurement is divided into hand palm length and finger length. Thereby scaling function in each axis of the tool-handle corresponds to a predetermined anthropometric value of the target population. The scaling in the z-axis accounts for the target population hand width, scaling in $\mathrm{x}$-axis accounts for the target population hand palm and finger length and scaling in y-axis accounts for finger length (Fig. 3). All three scaling factors are independent from each other and are computed based on target population anthropometric measurements. 


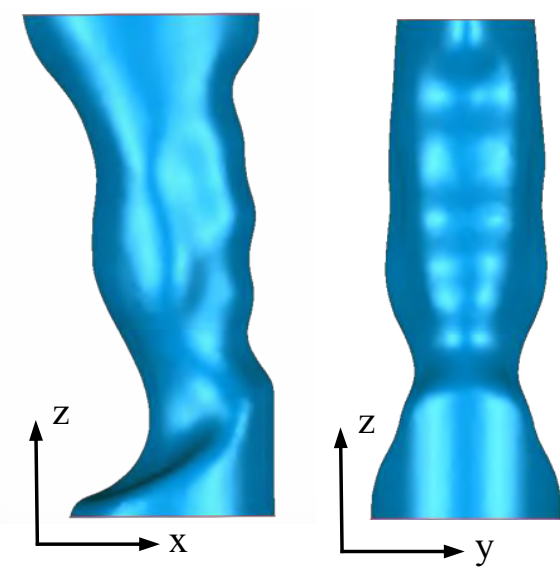

Figure 3: Sizing of the tool-handle for target populations.

\subsection{Factor of generality}

In order to obtain a generalized shape of the tool-handle, which can be used by wider target population and wider range of tasks, a factor of generality is introduced. This is a smoothing function with predetermined smoothing parameters, which allows local topological smoothing of the resulting symmetrical handle. The parameters are set to produce more generalized shape of a handle without any indentations and small topological details, which limit the use of the handle by broader population and wider range of tasks (Fig. 4).

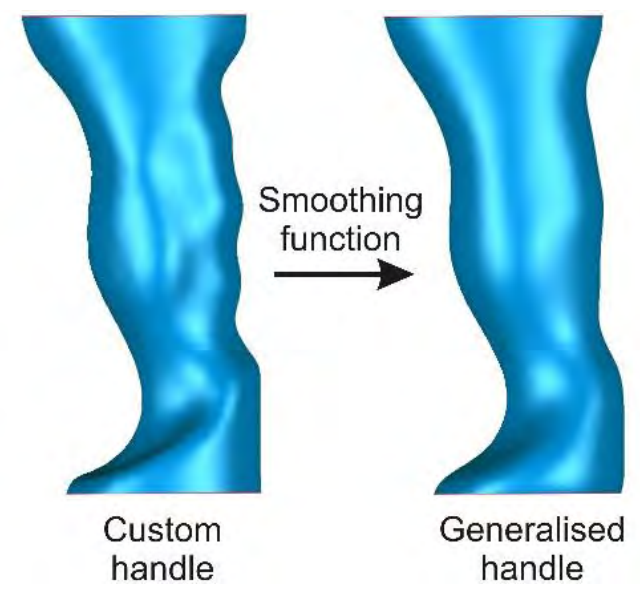

Figure 4: Custom handle and resulting generalized handle based on smoothing function.

Topological deviations of the generalized handle in comparison to the custom handle were also investigated. The histogram of the deviations shows that most of the topological deviations are concentrated around the deviation of about zero millimetres, which shows that despite the use of the smoothing function the basic shape has been retained (Fig. 5). Therefore also the effect of the higher contact pressure due to shape generalizations of the handle has been kept at lowest level.

\subsection{Tool-handle material recommendations based on FEM}

Tool-handle material has huge effect on the contact pressure values and its distribution, which can effect the development of various ATD and CTD. To present the possibility of reduction of contact pressure on fingertip finite element analysis as discussed in previous research was utilized [30]. 


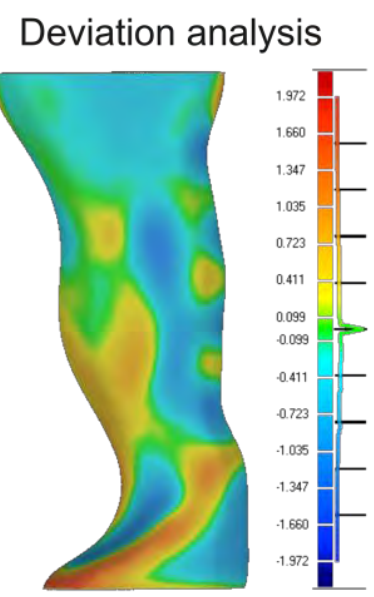

Statistics:

Average distance: $-0.058 \mathrm{~mm}$ positive: $0.320 \mathrm{~mm}$ negative: $-0.423 \mathrm{~mm}$ Std. dev.: $0.511 \mathrm{~mm}$

Figure 5: Topological deviation analysis of both handles.

The two-dimensional simulation from previous paper was extended into a threedimensional simulation of whole fingertip, where the fingertip geometry was modelled based on magnetic resonance imaging [42]. Various materials and composites were simulated. It has been shown that hyper-elastic foam materials, which take into account the non-linear behaviour of fingertip soft tissue, can lower the contact pressure whilst maintaining low deformation rate of the tool-handle material for maintaining sufficient rate of stability of the hand tool in the hands. Lower contact pressure also lowers the risk of ATD and CTD, and increases comfort whilst maintaining performance. The knowledge resulting from the simulations was then integrated into the system as an advisory module consisting of toolhandle material database and their mechanical behaviour. Contact pressures and their distributions while grasping resulting from the FE simulation were provided in diagrams and images (Fig. 6). Based on the results and the intended tool-handle use, the module suggests appropriate material combination to find a material with best compromise of lowering the overall and local contact pressure and maintaining high rate of stability of tool-handle in hands.
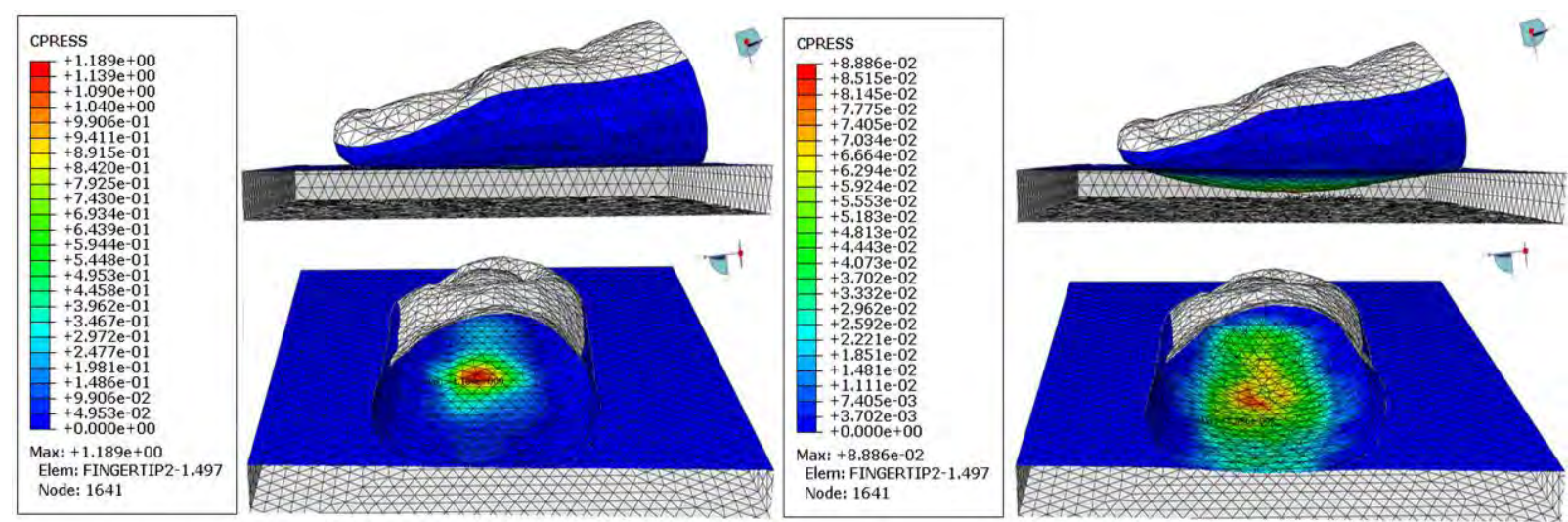

Figure 6: Contact pressure distribution and fingertip and tool-handle material deformation for steel (left) and composite of ethylene propylene diene monomer (EPDM) rubber and poly urethane (PU) foam (right) as tool-handle material. 


\subsection{Decision support system}

The resulting symmetrical tool-handle with the mathematical model for sizing generates optimal sized and shaped tool-handles for a target population, but it does not consist of basic ergonomic recommendations, which are also important in the design process of the hand tools and especially tool-handles. Therefore the mathematical model and the knowledge regarding correct tool-handle material determination was integrated into an existing intelligent decision support system OSCAR [31]. Within the system different classes are interconnected with various attributes and their values at the head of the rules in order to describe case specific situations, in which those recommendations for product design should be taken into consideration. The system consist of most important ergonomics design goals and respective design recommendations relating to hand tool design process from which to list just a few: dimensioning and configuration, wrist positioning, tissue compression, excessive forces reduction, etc. [31].

\section{RESULTS}

The result is a comprehensive intelligent decision support system, which consists of general design recommendations, a mathematical model for generating optimal sized and shaped toolhandles and recommendations for correct choice of too-handle materials. Based on the design requirements of the new hand tool, the designer has to determine the target population, which can be very specific or more general according to anthropometric measurements. Based on the target population, the system provides basic design recommendations, such as sizing, configuration, clearance, etc. Afterwards the anthropometric measurements, which are needed for optimal sized and shaped tool-handle are entered manually or selected from anthropometric databases. The mathematical module suggests a factor of generality based on the chosen target population, hand tool and task. The factor of generality is a smoothing function, which is used to smooth the handle generated with the mathematical model based on the anthropometric measurements for the chosen target population. Based on the boundary conditions, the system then generates the optimal sized and shaped tool-handle and also provides design recommendations, which can be directly used in the design process of new hand tool. Afterwards, the tool-handle material recommendation module suggests an appropriate tool-handle material combination in order to lower the contact pressure and maintain sufficient stability of the tool-handle in the hands.

\section{DISCUSSION}

Basic handle recommendations and existing mathematical models for diameter determination do not provide comprehensive aid in the design process of tool-handles; therefore nonexperienced designers are confronted with a challenging task. Nevertheless also the decision support systems do not guarantee the achievement of high ergonomic values in tool-handle design, since the design recommendations are mostly to loose and the end design is mostly dependent on the designer's experience and creativity. This is especially prominent in the shape determination in handle design process, where authors mostly consider cylindrical or elliptical handles. Therefore previously presented mathematical model for generating optimal sized and shaped tool-handles were integrated into the existing decision support system OSCAR. This way the system provides a comprehensive design aid from selecting the target population, basic design and ergonomic recommendations, to generating optimal sized and shaped tool-handles for the selected target population with correct material choice of the toolhandle. 
The correct shape determination is crucial, since it additionally improves the ergonomics of the handle. The accuracy of the proposed mathematical model is strongly dependent on used methods; therefore they have already been verified for the accuracy. Thereby it can be also assumed, that the maximum possible grip force can be exerted and subjective comfort rating, which is diameter dependent, is also maximized. Contact area of the tool-handles developed with the decision support system and its mathematical model has proved to increase the contact area of the handle by over $25 \%$ in comparison to an optimal cylindrical handle. This can be attributed to the anatomical handle shape, which is derived from the hand in its optimal power grasp posture. Thereby also the contact forces are spread over greater contact area, which lowers the overall contact pressure. This has proven to provide greater comfort rate and is therefore more likely to prevent ATD and CTD in comparison to a cylindrical handle.

Broader dissemination of DHMs and DHHMs is usually prevented due to their complex usage. Additionally, most of the dynamic DHHMs with autonomous grasping are intended for evaluating and analysing an existing CAD designs inside the virtual environment. Therefore designer has to possess extensive knowledge regarding size and shape of the tool-handle. The autonomous grasps generated by the DHHM are usually evaluated by the operator visually. Real-world grasping is very complex and is also depends on the subjective comfort rating of the user, therefore this kind of evaluation can be unreliable. Perceived subjective comfort using tool-handle is strongly correlated with user performance. It is therefore necessary to incorporate this aspect of product evaluation during the design phase, which is not possible when using DHHMs. Comfort is a subjectively-defined feeling that differs from person to person, therefore it cannot be predicted neither by objective methods (e.g. grip-force, contact pressure, electromyography, finite element analyses, etc.) nor by the resulting mathematical models that can only predict the physical aspects on the perceived comfort. Tool-handles based on decision support system can increase subjective comfort rating, resulting in increased human-product performance. The subjective comfort rating has been measured based on manufactured rapid prototypes of the corresponding handles and subjective comfortrating questionnaire. The tool-handle developed using mathematical model within the decision support system showed a significant increase in perceived subjective comfort rating. The increase of perceived subjective comfort rating is due to anatomical shape of the handle, since the handle considers optimal power grasp posture in the development of the handle. Based on this, the handle provides best fit to the target population.

If the produced autonomous grasp of the existing DHHMs is feasible and realistic, ergonomics design errors can be identified and design solutions can be proposed. However this always requires extensive ergonomic knowledge and experience. This iterative design process is repeated until the design does not meet the desired level of ergonomics. On the other hand, direct development of tool-handles with developed decision support system generates optimal sized and shaped too-handles. Therefore physical prototypes for the purpose of performing ergonomic analyses become unnecessary. This results in increased time efficiency and tool-handles with improved ergonomics, without the need for comprehensive knowledge of ergonomics by the designer.

It has been shown that there is even less knowledge and also experience among designers regarding the correct choice for the material of the tool-handle, which will be in direct contact with the hand. Designers are used to most common and known materials such as plastics, wood, steel and rubber, however they do not possess knowledge of the physical response of human hand to these materials while grasping. Additionally, comprehensive knowledge of more complex materials, such as hyper-elastic foams, which have proven to lower the overall and local contact pressures, is low or non-existent. Therefore existing general material choice knowledge and also knowledge obtained from previous performed finite element analyses 
was integrated into the advisory module for tool-handle material choice. Additionally the FE model is provided in the module for designers who wish to perform analyses with materials of their own. The module for tool-handle material recommendations provides the designers simple aid in the material choice to lower the overall and also local contact pressure and therefore also lower the risk for pressure dependent ATD and CTD. Correct tool-handle material determination therefore additionally improves the ergonomics of the tool-handle.

It has been shown that it is possible to design tool-handles without any prior ergonomic knowledge with the developed decision support system, since the designer is provided with needed design and material recommendations and mathematical model for generating the optimal sized and shaped tool-handle for target population. With the use of the decision support system and especially the mathematical model, the designing process time is reduced, which was also shown in several preliminary tests of the system. Following improved product ergonomics, the market value of the product is increased, thus enhancing the competitiveness of the product on the market.

\section{CONCLUSION}

Previously developed methods, which consider also the anatomical shape of the hand in development of the optimal shaped handle, were used and integrated into a decision support system for generating optimal sized and shaped tool-handle for a target population and task. The proposed intelligent decision support system provides comprehensive aid in ergonomic and aesthetic product design process in form of recommendations and in generation of optimal sized and shaped tool-handles. Additionally, the system provides aid to determine appropriate tool-handle material in order to lower the contact pressure. Thereby small companies, which do not employ an ergonomist, can develop hand tools considering ergonomic principles for improved performance, comfort and avoidance of acute and cumulative trauma disorders in shorter time.

Future work should also revise the factor of generality in order to provide a more precise amount of smoothing. The smoothing function is applied to whole handle, although future work should also consider the fact that different surface parts of the handle require different quantity of smoothing to achieve the desired generality. The advisory module for tool-handle material choice should be further extended with more material choices. The decision support system should be also further tested to evaluate and analyse the impact on the hand tool design process.

\section{REFERENCES}

[1] Hogberg, D.; Backstrand, G.; Lamkull, D.; Hanson, L.; Ortengren, R. (2008). Industrial customisation of digital human modelling tools, International Journal of Services Operations and Informatics, Vol. 3, No. 1, 53-70, doi:10.1504/IJSOI.2008.017705

[2] Yakou, T.; Yamamoto, K.; Koyama, M.; Hyodo, K. (1997). Sensory evaluation of grip using cylindrical objects, JSME International Journal Series C, Mechanical Systems, Machine Elements and Manufacturing, Vol. 40, No. 4, 730-735, doi:10.1299/jsmec.40.730

[3] Hall Jr., N. B.; Bennett, E. M. (1956). Empirical assessment of handrail diameters, Journal of Applied Psychology, Vol. 40, No. 6, 381-382, doi:10.1037/h0042873

[4] Amis, A. A. (1987). Variation of finger forces in maximal isometric grasp tests on a range of cylinder diameters, Journal of Biomedical Engineering, Vol. 9, No. 4, 313-320, doi:10.1016/ 0141-5425(87)90079-3

[5] Chen, Y. (1991). An evaluation of hand pressure distribution and forearm flexor muscle contribution for a power grasp on cylindrical handles, Ph.D. Dissertation, University of Nebraska 
[6] Sancho-Bru, J. L.; Giurintano, D. J.; Perez-Gonzalez, A.; Vergara, M. (2003). Optimum tool handle diameter for a cylinder grip, Journal of Hand Therapy, Vol. 16, No. 4, 337-342, doi:10.1197/S0894-1130(03)00160-1

[7] Grant, K. A.; Habes, D. J.; Steward, L. L. (1992). An analysis of handle designs for reducing manual effort: The influence of grip diameter, International Journal of Industrial Ergonomics, Vol. 10, No. 3, 199-206, doi:10.1016/0169-8141(92)90033-V

[8] Oh, S.; Radwin, R. G. (1993). Pistol grip power tool handle and trigger size effects on grip exertions and operator preference, Human Factors, Vol. 35, No. 3, 551-569, doi:10.1177/ $\underline{001872089303500311}$

[9] Johnson, S. L. (1993). Ergonomic Hand Tool Design, Hand Clinics, Vol. 9, No. 2, 299-311

[10] Blackwell, J. R.; Kornatz, K. W.; Heath, E. M. (1999). Effect of grip span on maximal grip force and fatigue of flexor digitorum superficialis, Applied Ergonomics, Vol. 30, No. 5, 401-405, doi:10.1016/S0003-6870(98)00055-6

[11] Garneau, C. J.; Parkinson, M. B. (2009). Optimization of tool handle shape for a target user population, Proceedings of the ASME International Design Engineering Technical Conferences and Computers and Information in Engineering Conference, 1029-1036

[12] Seo, N. J.; Armstrong, T. J. (2008). Investigation of grip force, normal force, contact area, hand size, and handle size for cylindrical handles, Human Factors, Vol. 50, No. 5, 734-744, doi:10.1518/001872008X354192

[13] Ayoub, M. M.; Presti, P. L. (1971). The determination of an optimum size cylindrical handle by use of electromyography, Ergonomics, Vol. 14, No. 4, 509-518, doi:10.1080/ 00140137108931271

[14] Kong, Y.-K.; Lowe, B. D. (2005). Optimal cylindrical handle diameter for grip force tasks, International Journal of Industrial Ergonomics, Vol. 35, No. 6, 495-507, doi:10.1016/j.ergon. 2004.11.003

[15] Wu, J. Z.; Dong, R. G. (2005). Analysis of the contact interactions between fingertips and objects with different surface curvatures, Proceedings of the Institution of Mechanical Engineers, Part H: Journal of Engineering in Medicine, Vol. 219, No. 2, 89-103, doi:10.1243/095441105X9327

[16] Wu, J. Z.; Cutlip, R. G.; Andrew, M. E.; Dong, R. G. (2007). Simultaneous determination of the nonlinear-elastic properties of skin and subcutaneous tissue in unconfined compression tests, Skin Research and Technology, Vol. 13, No. 1, 34-42, doi:10.1111/j.1600-0846.2007.00182.x

[17] Rossi, J.; Berton, E.; Grelot, L.; Barla, C.; Vigouroux, L. (2012). Characterisation of forces exerted by the entire hand during the power grip: effect of the handle diameter, Ergonomics, Vol. 55, No. 6, 682-692, doi:10.1080/00140139.2011.652195

[18] Gurram, R.; Rakheja, S.; Gouw, G. J. (1995). A study of hand grip pressure distribution and EMG of finger flexor muscles under dynamic loads, Ergonomics, Vol. 38, No. 4, 684-699, doi:10.1080/00140139508925140

[19] Eksioglu, M. (2004). Relative optimum grip span as a function of hand anthropometry, International Journal of Industrial Ergonomics, Vol. 34, No. 1, 1-12, doi:10.1016/j.ergon. 2004.01.007

[20] Welcome, D.; Rakheja, S.; Dong, R.; Wu, J. Z.; Schopper, A. W. (2004). An investigation on the relationship between grip, push and contact forces applied to a tool handle, International journal of Industrial Ergonomics, Vol. 34, No. 6, 507-518, doi:10.1016/j.ergon.2004.06.005

[21] Aldien, Y.; Welcome, D.; Rakheja, S.; Dong, R.; Boileau, P.-E. (2005). Contact pressure distribution at hand-handle interface: role of hand forces and handle size, International Journal of Industrial Ergonomics, Vol. 35, No. 3, 267-286, doi:10.1016/j.ergon.2004.09.005

[22] Fellows, G. L.; Freivalds, A. (1991). Ergonomics evaluation of a foam rubber grip for tool handles, Applied Ergonomics, Vol. 22, No. 4, 225-230, doi:10.1016/0003-6870(91)90225-7

[23] Seo, N. J.; Armstrong, T. J. (2011). Effect of elliptic handle shape on grasping strategies, grip force distribution, and twisting ability, Ergonomics, Vol. 54, No. 10, 961-970, doi:10.1080/ $\underline{00140139.2011 .606923}$

[24] Dolsak, B.; Novak, M. (2011). Intelligent decision support for structural design analysis, Advanced Engineering Informatics, Vol. 25, No. 2, 330-340, doi:10.1016/j.aei.2010.11.001

[25] Rieg, F.; Hackenschmidt, R.; Alber-Laukant, B. (2012). Finite Elemente Analyse für Ingenieure, Carl Hanser Verlag, München, doi:10.3139/9783446434691 
[26] Krajnc, M.; Dolsak, B. (2013). Computer and experimental simulation of biomass production using drum chipper, International Journal of Simulation Modelling, Vol. 12, No. 1, 39-49, doi:10.2507/IJSIMM12(1)4.223

[27] Coelho, R. M.; Alves de Sousa, R. J.; Fernandes, F. A. O.; Teixeira-Dias, F. (2013). New composite liners for energy absorption purposes, Materials \& Design, Vol. 43, 384-392, doi:10.1016/j.matdes.2012.07.020

[28] Wu, J. Z.; Welcome, D. E.; Krajnak, K.; Dong, R. G. (2007). Finite element analysis of the penetrations of shear and normal vibrations into the soft tissues in a fingertip, Medical Engineering \& Physics, Vol. 29, No. 6, 718-727, doi:10.1016/j.medengphy.2006.07.005

[29] Wu, J. Z.; Wimer, B. M.; Welcome, D. E.; Dong, R. G. (2012). An analysis of contact stiffness between a finger and an object when wearing an air-cushioned glove: The effects of the air pressure, Medical Engineering \& Physics, Vol. 34, No. 3, 386-393, doi:10.1016/j.medengphy. 2011.08.004

[30] Harih, G.; Dolsak, B. (2014). Recommendations for tool-handle material choice based on finite element analysis, Applied Ergonomics, Vol. 45, No. 3, 577-585, doi:10.1016/j.apergo.2013. $\underline{07.014}$

[31] Kaljun, J.; Dolsak, B. (2012). Ergonomic design knowledge built in the intelligent decision support system, International Journal of Industrial Ergonomics, Vol. 42, No. 1, 162-171, doi:10.1016/j.ergon.2011.11.009

[32] Kaljun, J.; Dolsak, B. (2004). Computer aided ergonomic and aesthetic design, Proceedings of the $8^{\text {th }}$ International Design Conference DESIGN 2004, 1081-1086

[33] Pena-Pitarch, E.; Yang, J.; Abdel-Malek, K. (2009). Virtual human hand: grasping and simulation, Duffy, V. G. (Ed.), Digital Human Modeling (Lecture Notes in Computer Science, Vol. 5620), Springer-Verlag, Berlin, 140-149

[34] Endo, Y.; Kanai, S.; Kishinami, T.; Miyata, N.; Kouchi, M.; Mochimaru, M. (2007). A computeraided ergonomic assessment and product design system using digital hands, Duffy, V. G. (Ed.), Digital Human Modeling (Lecture Notes in Computer Science, Vol. 4561), Springer-Verlag, Berlin, 833-842

[35] Harih, G.; Dolsak, B. (2013). Tool-handle design based on a digital human hand model, International Journal of Industrial Ergonomics, Vol. 43, No. 4, 288-295, doi:10.1016/j.ergon. 2013.05.002

[36] Kaljun, J.; Dolsak, B. (2012). Improving products' ergonomic value using intelligent decision support system, Strojniski vestnik - Journal of Mechanical Engineering, Vol. 58, No. 4, 271-280, doi:10.5545/sv-jme.2011.193

[37] Kaljun, J.; Obad, M.; Dolsak, B. (2011). Intelligent decision support system for ergonomic product design, Strojarstvo, Vol. 53, No. 3, 221-230

[38] Kaljun, J.; Dolsak, B. (2012). Ergonomic design recommendations based on an actual chainsaw design, South African Journal of Industrial Engineering, Vol. 23, No. 2, 215-229, doi:10.7166/ 23-2-342

[39] Harih, G.; Cretnik, A. (2013). Interdisciplinary approach to tool-handle design based on medical imaging, BioMed Research International, Vol. 2013, Article ID 159159, 8 pages, doi:10.1155/2013/159159

[40] Gordon, C. C.; Churchill, T.; Clauser, C. E.; Bradtmiller, B.; McConville, J. T.; Tebbetts, I.; Walker, R. A. (1989). 1988 anthropometric survey of U. S. Army personnel: Summary statistics interim report, Technical Report NATICK/TR-89/027, U. S. Army Natick Research, Development \& Engineering Center, Natick, Massachusetts

[41] Garneau, C. J.; Parkinson, M. B. (2012). Optimization of product dimensions for discrete sizing applied to a tool handle, International Journal of Industrial Ergonomics, Vol. 42, No. 1, 56-64, doi:10.1016/j.ergon.2011.08.005

[42] Yoshida, H.; Tada, M.; Mochimaru, M. (2011). A study of frictional property of the human fingertip using three-dimensional finite element analysis, MCB: Molecular \& Cellular Biomechanics, Vol. 8, No. 1, 61-72, doi:10.3970/mcb.2011.008.061 